\title{
TEMPORARY WAFER BONDING AND DEBONDING BY AN ELECTROCHEMICALLY ACTIVE POLYMER ADHESIVE FOR 3D INTEGRATION
}

\author{
Hithesh K Gatty*, Frank Niklaus, Göran Stemme and Niclas Roxhed \\ Micro and Nanosystems, KTH Royal Institute of Technology, Stockholm, Sweden
}

\begin{abstract}
Thin wafer handling is an important issue in $3 \mathrm{D}$ integration technologies. This paper reports on an efficient method for bonding a thin wafer and debonding it at room temperature from a carrier wafer. This method addresses the major problem of fragility and flexibility in handling of thin wafers used in TSV fabrication. In the presented method the carrier wafer is spin coated with an electrochemically active polymer adhesive. It is then bonded to a device wafer. The wafer stack is thinned and finally released from the carrier wafer by applying a voltage.
\end{abstract}

\section{INTRODUCTION}

Temporary bonding of thin silicon wafers $(<100 \mu \mathrm{m})$ is necessary for example in making through silicon vias (TSV's). Thin silicon wafers are fragile and difficult to handle due to their increased flexibility. A reliable handling solution is needed to overcome the challenges of thin wafer handling. One possibility is by attaching the thin device wafer to a carrier wafer using wafer bonding. There are various methods of attaching two wafers. One of the methods is by applying adhesive on a carrier wafer and bonding it with a device wafer using a wafer bonder. Several different polymers have been studied for use in adhesive bonding; including epoxies, thermoplastic adhesives and photoresist. Polymers that are used as adhesives for wafer bonding were previously experimentally investigated for bond strength at low temperature $[1,2]$.

For temporary wafer handling, both bonding and debonding properties of the adhesives are important. Efficient bonding and debonding methods are needed to overcome the problem of fragility and flexibility while handling thin wafers. Two methods are predominantly applied in temporary wafer bonding and releasing of thin wafers in semiconductor industry. In the first method, an adhesively bonded wafer stack is heated to an elevated temperature during which the adhesive softens and becomes liquid $[3,4]$. The device wafer is then released by sliding it off from the carrier wafer. This method has drawbacks since an elevated temperature $\left(>200^{\circ} \mathrm{C}\right)$ is needed for debonding. The other method is the solvent based debonding e.g. Zone bonding technique [5]. However, this method requires edge treatment of the adhesive on the wafer to create two zones of different adhesive strength and requires many processing steps before the actual release of the carrier wafer.

In this paper, we present a novel approach for bonding and releasing thin wafers without having to apply elevated temperature or solvent based treatment of the adhesive. The debonding principle is based on ionic conduction in the adhesive leading to release of the device wafer. This type of temporary wafer handling proves advantageous in addressing the major problem of fragility and flexibility of handling thin wafers. The process flow is developed for spin coating the adhesive on the carrier wafer and the debonding forces before and after applying a voltage to the stack is investigated. The current density w.r.t time for debonding is also investigated.

\section{CONCEPT}

In this work the bonding and debonding for temporary wafer handling of a thin wafer is described. It is done with the help of modified electrochemically active polymer that is commercially sold as Electrelease ${ }^{\mathrm{TM}} \mathrm{H} 23$ [6]. The ElectRelease adhesive was originally developed for bonding and debonding of aluminum parts as illustrated in Figure 1. The adhesive bond is made of electrochemically debondable composition that has matrix functionality and electrolytic functionality. The matrix functionality provides an adhesive bond between the wafers while the electrolytic functionality provides sufficient ionic conductivity to the composition to support a Faradaic reaction at the interface. One of the causes of debonding is the oxidation of the anode surface leading to the formation of oxide [7].



Before applying voltage



After applying voltage
Figure 1: Concept of the debonding technique using the ElectRelease adhesive.

The viscosity of the commercially available adhesive formulation is high and cannot be used in microfabrication processes like spin coating. Hence the adhesive must be diluted in order to reduce the viscosity. Cyclopentanone (CPT) was found to be a suitable solvent for this type of electrochemically active polymers as it does not destroy the electrical and chemical properties of the polymer. An investigation is carried out in order to find the ratio of mixing and the spin speed that is required to uniformly coat the wafer. The adhesive is a two part mixture where one part is the base or 'resin' and the other the hardener or 'activator'. Two different mixture ratios were evaluated for viscosity by spin coating on a wafer. The first mixture ratio was 59.7 wt \%: 10.5 wt \%: 29.8 wt \% (resin: hardner: CPT, respectively). This ratio was found to be 'watery' and was 
not suitable for spin coating the adhesive on a wafer. Hence the CPT solvent was reduced by $50 \%$ and its viability for spin coating was evaluated on the carrier wafer. This ratio was found to be stable and it was possible to spin coat the adhesive on the carrier wafer.

The adhesive with this mixture ratio is used for further investigation. Figure 2 shows the graph of the thickness of the adhesive in relation to spin speeds at different mixture ratios. For each spin speed the thickness of the adhesive was measured at multiple points on the wafer. The thickness variation was measured to be approximately $10 \%$ across the wafer. Bonding of the device and carrier wafer is carried out after spin coating of the adhesive on the carrier wafer followed by thinning down of the device wafer. The debonding is demonstrated by applying a voltage between the carrier and the device wafer. The following section describes the fabrication process.

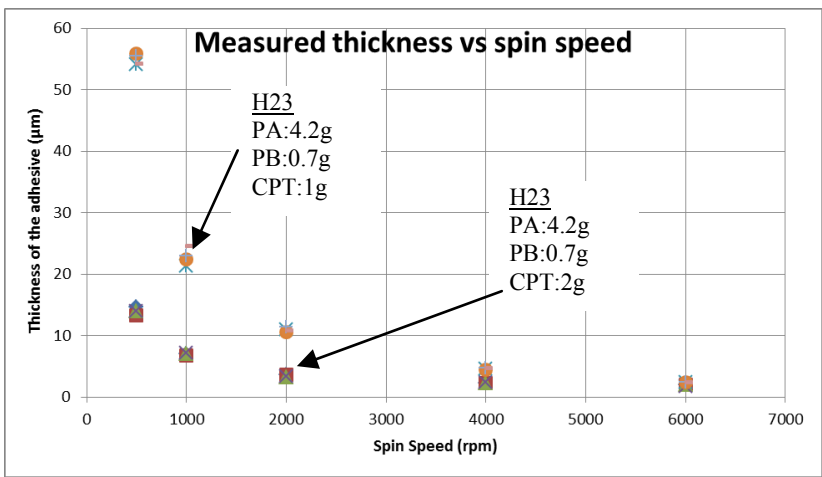

Figure 2: Plot showing the thickness vs spinspeed of the diluted ElectRelease adhesive. Thickness is measured at multiple points on the wafer for each spin speed.

\section{FABRICATION}

To demonstrate the ability to handle thin wafers while processing, monocrystaline silicon of $100 \mathrm{~mm}$ diameter wafer of thickness $100 \mu \mathrm{m}$ is used as the device wafer. The carrier wafer is a $550 \mu \mathrm{m}$ thick $\mathrm{p}+$ doped silicon wafer.

Diluted ElectRelease (ER) adhesive is spun on the carrier wafer using an OPTIspin SST20 spinner. The wafer is spun at $2000 \mathrm{rpm}$ (figure 3(a)). The uniformity of the adhesive is evaluated using a surface profiler Tencor-P10 and found to yield a uniform thickness of $11 \mu \mathrm{m}$ having < $10 \%$ thickness variation over the wafer. The device wafer is sputtered on one side with a $250 \mathrm{~nm}$ thick aluminum layer. The exposed aluminum area on the device wafer is used as the contact for applying a voltage to the top electrode (anode) as shown in figure 3(b).

Once the adhesive is spin coated on the wafer, it has a settling time of 6 min within which the following bonding step has to be carried out before the adhesive solidifies. During this period, the aluminum coated device wafer is manually aligned with the carrier wafer (figure 3(b)). After the device wafer is aligned with the carrier wafer, the stack is covered with a clean room tissue paper on both the sides. This is done to absorb excessive adhesive that is pressed out from the wafer stack during bonding. Graphite sheets with a thickness of $500 \mu \mathrm{m}$ are placed above the tissue paper on both sides of the stack as shown in figure 3(c). The graphite sheet act as compliant chucks, evening out pressure non-uniformities ensuring that a uniform load is

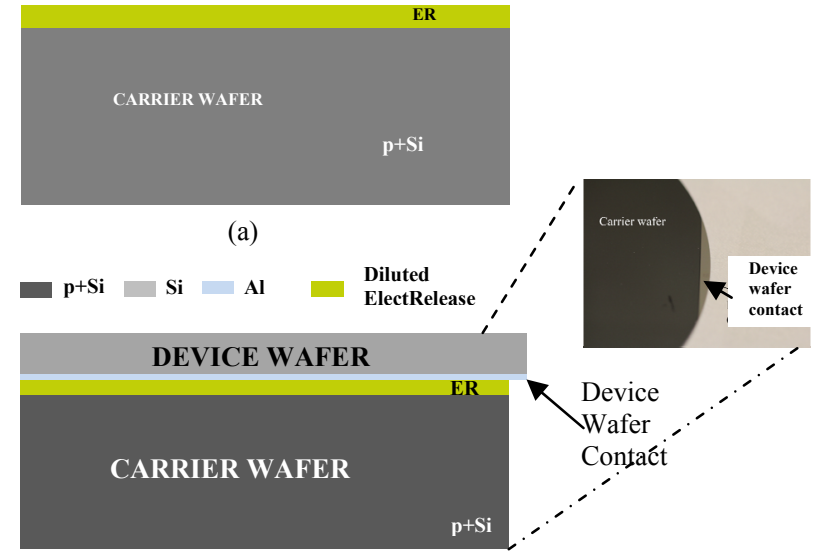

(b)

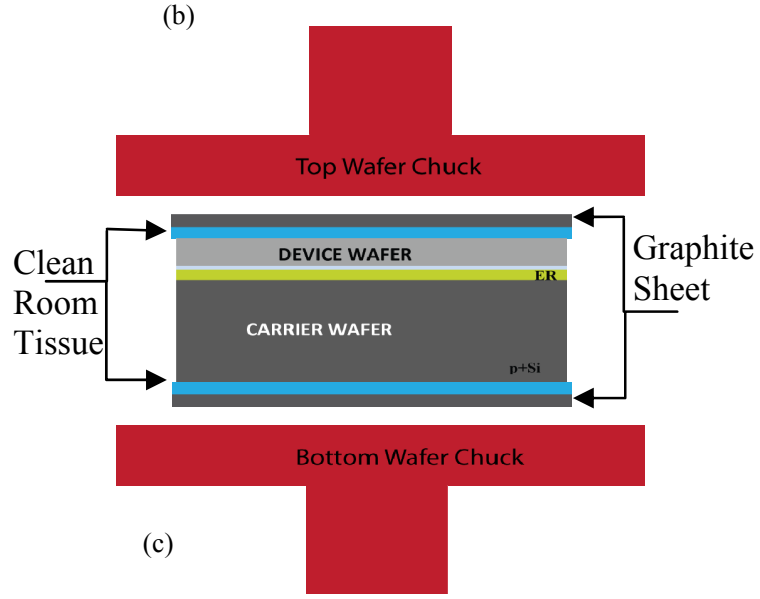

Figure 3(a-c): 3(a) Spin coating of the carrier wafer with ElectRelease(ER) adhesive. 3(b) Illustration showing wafer alignment of $100 \mu \mathrm{m}$ thick device wafer that is coated with a $250 \mathrm{~nm}$ thick aluminum layer. Inset shows the image of the contact area. 3(c) Wafer stack showing the assembly of clean room tissue and graphite sheets.

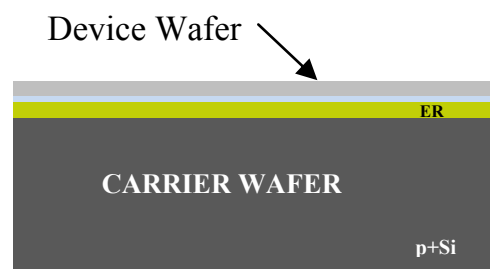

Figure 4: The device wafer is thinned down to approximately $40 \mu \mathrm{m}$ by etching in $\mathrm{SF}_{6}$ plasma.

spread across the stack.

The wafer stack is transferred to a Suss Microtec CB8 wafer bonder. Once the pressure is pumped down to $10^{-4}$ mbar, a bonding force of about $1 \mathrm{kN}$ is applied on the stack at a temperature of $25^{\circ} \mathrm{C}$ for $20 \mathrm{~min}$. After this period the temperature is ramped up to $80^{\circ} \mathrm{C}$ with a ramp up time of 5 min. The wafer stack is held at this temperature inside the chamber for $30 \mathrm{~min}$ while maintaining the force. During this period the adhesive undergoes curing and hardens, which results in bonding of the device wafer to the carrier wafer. Alternatively, curing of the adhesive can also be done at room temperature, which takes about 22-24hrs. 
The wafer stack is then transferred to a STS ICP DRIE etcher and the device wafer is thinned down to approximately $40 \mu \mathrm{m}$ (figure 4). It is etched in the ICP chamber for $25 \mathrm{~min}$. During this period the adhesive properties were observed for influence of temperature, and its tolerance to plasma. It is found that the wafer stack remained intact without any visible defects after the process.

\section{EXPERIMENTS AND RESULTS}

To test the debonding feature of the adhesive, two main experiments were conducted. Firstly, an experiment pertaining to the measurement of bond strength before and after application of a voltage is conducted. Secondly, an experiment relating to decaying current density that leads to debonding of the device wafer is carried out. The wafer stack is fixated onto an Al plate of thickness approximately $3 \mathrm{~mm}$. This provided not only an electrical contact to the carrier wafer but also facilitated in handling the carrier wafer for testing of the bond strength without handling of the wafer stack. To allow handling of the device wafer, a UV release tape is attached on the device wafer.

A dynamometer was used to measure the bond strength of the wafer stack. It is fixed at one of the corners of the UV tape and was pulled upwards so that the UV tape also pulls the device wafer as shown in figure 5 .

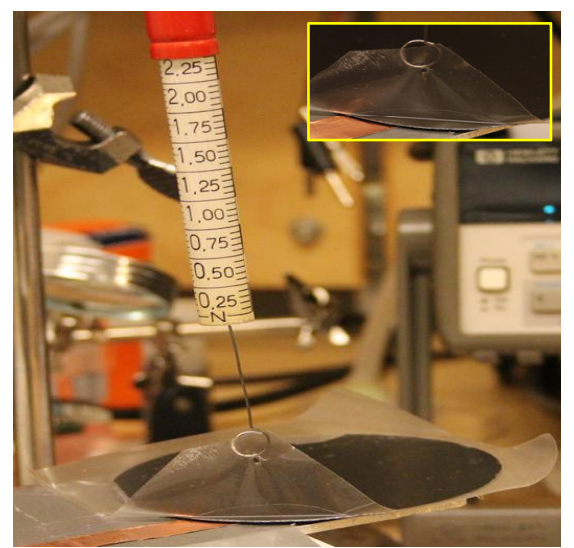

Figure 5: Image showing the application of force to test the bond strength of the stack. Inset image shows peeling of the UV tape from the device wafer.

A force of approximately $2.25 \mathrm{~N}$ was measured with the dynamometer. At this force the UV tape starts to peel off from the device wafer, indicating that the device wafer is attached with higher bond strength to the carrier wafer than the UV tape to device layer. The strength of the bond is also tested by using a scalpel that is carefully inserted between the device and the carrier wafers, and twisted which led to breakage of the stack.



Figure 6: The UV release tape is attached to the device wafer and a voltage is applied between device and carrier wafer.
Debonding is carried out by applying a voltage between the carrier wafer and the device wafer. The device wafer is at a positive potential and the debonding takes place at the anodic interface that releases the device wafer from the stack leaving the adhesive on the carrier wafer. To realize debonding, a constant voltage of $+50 \mathrm{~V}$ is applied between the carrier (cathode) and device (anode) wafers as shown in Figure 6. Once the current density falls to a low value $(\sim 1$ $\left.\mathrm{mA} / \mathrm{cm}^{2}\right)$, the voltage is turned off. The bond force is again measured using a dynamometer by carefully pulling the UV tape from one side of the wafer stack. The force was measured to be approximately $0.5 \mathrm{~N}$. At this force the device wafer starts to peel off from the carrier wafer indicating low bond strength. This low debonding strength aids in quick and effortless release of the carrier wafer as shown in figure 7.

Using the same fabrication and evaluation procedure another set of wafers were prepared to investigate debonding relating to the current density and time. These experiments showed that after applying a voltage to the stack, the ionic current increases to about $11 \mathrm{~mA} / \mathrm{cm}^{2}$; after which, the current density falls to a low value (approx. 1 $\mathrm{mA} / \mathrm{cm}^{2}$ ) in approximately $42 \mathrm{~s}$ as shown in figure 8 . Once the current density has stabilized on a low value, the voltage is turned off. To further demonstrate the debonding efficiency, the device wafer is manually released from the carrier wafer by applying a gentle force on the UV tape and peeling it radially inwards as shown in figures 9 and 10 .


Figure 7: Schematic process of debonding and an image of debonding and measurement of force after applying a voltage.

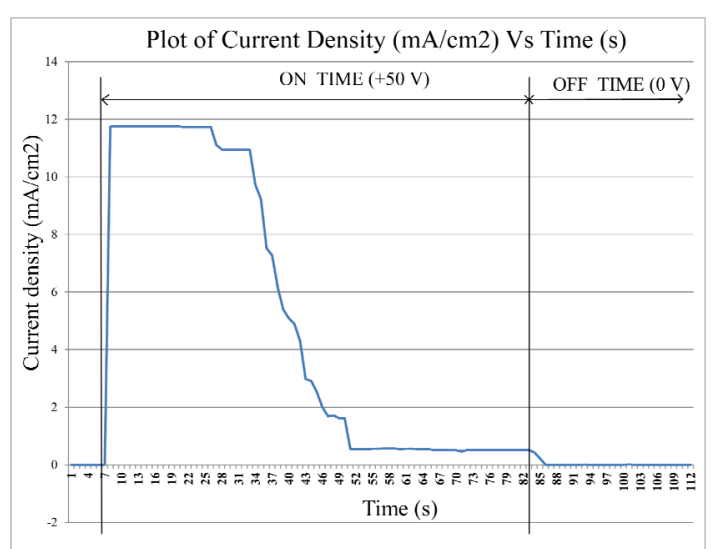

Figure 8: Plot showing the measured current density when $50 \mathrm{~V}$ is applied to the wafer stack. 




Figure 9: Image showing the debonding of thin device wafer from the stack.

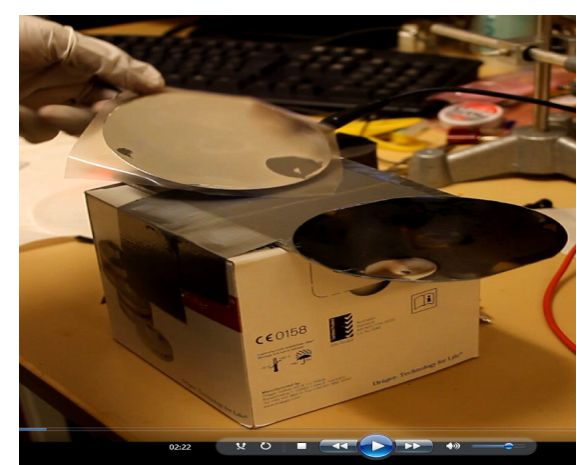

Figure 10: Image showing the device wafer release from the carrier wafer after debonding.

To evaluate the effect of joule heating, the temperature rise was simulated using COMSOL ${ }^{\mathrm{TM}}$ and it was found that a temperature increase of about $7-8^{\circ} \mathrm{C}$ can be expected. This temperature rise should not influence the debonding process. No signs of the cracking of the device wafer were observed as shown in figure 11. Further processing steps might include removing of UV tape by applying UV light on the device wafer and using it for subsequent processing step such as, TSV formation.

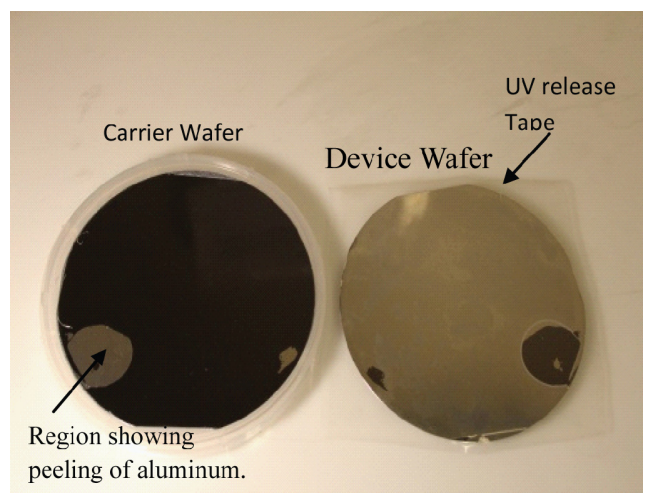

Figure 11: Image showing the device wafer after debonding. No cracking of the wafer is observed.

\section{CONCLUSIONS}

An efficient method for temporary wafer bonding and debonding using polarization of the adhesive at room temperature is demonstrated. The bonding force between the device and the carrier wafer is measured to be approximately $2.25 \mathrm{~N}$ before polarizing. The device wafer is released from the stack with a low force of $<0.5 \mathrm{~N}$ by applying a voltage of $50 \mathrm{~V}$. The device wafer thus released can be cleaned and used for further processing. This method of temporary handling of the wafer avoids heating of the adhesive and it does not require any solvent based treatment.

\section{ACKNOWLEDGMENT}

This work has been funded by the European Research Council (ERC) through the Advanced Grant (267528): Towards Cost-Efficient Flexible Heterogeneous Integration for Micro- and Nanosystem Fabrication. The author would also like to thank Nils and Hans Backmark foundation (EK2012-0047) for providing travel stipend to present the work at a conference.

\section{REFERENCES}

[1] F. Niklaus et al "Adhesive wafer bonding", J. Applied Physics 99, 031101 (2006)

[2] F.Niklaus et al "Low temperature full wafer adhesive bonding of structured wafers", Sensors \& Actuators A 92 (2001) 235-241.

[3] S. Pargfrieder et al, "Temporary Bonding and DeBonding Enabling TSV Formation and 3D Integration for Ultra-thin Wafers." EPTC conference 2008, 1301-1305.

[4] Rama Puligadda et al, "High-Performance Temporary Adhesives for Wafer Bonding Applications" Mater.Res.Soc. Symp. Proc. Vol. 970

[5] Alain Phommahaxay et al, "Ultrathin Wafer Handling in 3D Stacked IC Manufacturing Combining a Novel ZoneBOND ${ }^{\mathrm{TM}}$ Temporary Bonding Process with Room Temperature Peel Debonding", 3D systems Integration conference (3DIC) 2012, 1-4.

[6] www.exonera.com /Electrelease H23.

[7] S Leijonmarck et al, "Electrolytically assisted debonding of adhesives:An experimental investigation" J.Adhesion \& Adhesives 32 (2012) $39-45$

\section{CONTACT}

*H.K.Gatty, tel: +46760790758; hithesh@kth.se 